\title{
Data Analysis of Single Nucleotide Polymorphism in Human AGT Gene Using Computational Approach
}

\author{
Mohammed Youssif Mohammed, Afra Mohamed Al Bkrye, Hind Abdelaziz Elnasri, \\ Mona Abdelrahman Mohamed Khaier* \\ Department of Molecular Biology and Bioinformatics, College of Veterinary Medicine, University of Bahri, Khartoum, Sudan
}

Email address:

munakhaier@gmail.com (M. A. M. Khaier)

${ }^{*}$ Corresponding author

\section{To cite this article:}

Mohammed Youssif Mohammed, Afra Mohamed Al Bkrye, Hind Abdelaziz Elnasri, Mona Abdelrahman Mohamed Khaier. Data Analysis of Single Nucleotide Polymorphism in Human AGT Gene Using Computational Approach. International Journal of Genetics and Genomics. Vol. 8, No. 2, 2020, pp. 78-84. doi: 10.11648/j.ijgg.20200802.14

Received: January 10, 2020; Accepted: January 27, 2020; Published: April 7, 2020

\begin{abstract}
Background: The $A G T$ gene is gene responsible for regulation of protein called angiotensinogen which regulates blood pressure and balances fluids in the body. Hypertension happens due to many causes one of this is the defect in $A G T$ gene. Hypertension usually has no symptoms. However, it is a major risk factor for heart diseases, stroke, kidney failure, and eye problems. Objectives: in this study we use software to analyze the gene using different software and represented statistically and to detect the SNPs that can cause the disease. Material and Method: In this analysis using many software tools that can analyze the nsSNPs retrieved from NCBI website. These software include SIFT, I-mutant, Polyphen-2, PHD SNP and SNP\& Go, Projecthop and GeneMANIA. Results: The study showed that from 172 nsSNPs only 46 nsSNPs were deleterious while 126 were tolerated using SIFT. Two were benign, 11 were possibly damaging and 33 were probably damaging by Polyphen-2. Using Provean, 19 nsSNPs were neutral and 27 were deleterious. For PHD-SNP software 20 nsSNPs were disease related and 18 were neutral. Also SNPs were checked using SNP \& Go software that showed 32 neutral nsSNPs and 14 nsSNPs were disease associated variants. Using I-Mutant software 13 nsSNPs increase the stability of the protein and 33 decrease the protein stability. Conclusions: In conclusion, extensive functional and structural analyses are carried out to predict potentially damaging and deleterious nsSNPs of $A G T$ gene using bioinformatics and computational methods. In the study, 14 high confidence damaging nsSNPs are identified from $172 \mathrm{nsSNPs}$. Although bioinformatics tools have their limitations, the results from the present study may be convenient in future for further population based research activities and towards development of accuracy medicines.
\end{abstract}

Keywords: $A G T$ Gene, Hypertension, I-mutant, SIFT, SNP \& Go and PHD, Polyphen-2, Provean and Project Hope, SNP

\section{Introduction}

AGT is a gene use to control type of protein called angiotensinogen, which represent a part of angiotensin system, that function to regulate blood pressure and fluid in the body [1]. This regulation happens by converting angiotensinogen into angiotensin I [1]. And angiotensin I is converted into angiotensinogen II, that cause blood vessels to narrow leading to increase in blood pressure [2]. Also angiotensinogen II induce production of aldosterone hormone [2], which plays a role in salt absorption by kidney [1], leading to increase body fluids hence increase in blood pressure [3]. Normal blood pressure is important during fetal life which delivers oxygen to body tissue, also need it for kidney development especially the proximal tubules and growth factors involving in kidney structure. [4].

Many health conditions associated with the disease were caused by mutation in $A G T$ gene, among these is hypertension, a specific mutation in $A G T$ gene causes the disease [5]. However, hypertension is a major risk factor for heart disease, stroke, kidney failure, and eye problems. When blood pressure is elevated, the heart and arteries have to work harder than normal to pump blood through the body [6]. The extra work thickens the muscles of the heart and arteries and hardens or damages artery walls [7]. As a result, the flow of blood and oxygen to the heart and other organs is reduced. 


\section{Material and Methods}

Data retrieval: this was done using the dbSNP (http://www.ncbi.nlm.nih.gov/SNP/). Information regarding SNPs of $A G T$ gene was obtained during the year 2019. Interaction of this gene with other genes was investigated using GeneMANIA. Functional effect of the nsSNPs on the protein was investigated using SIFT, Polyphen-2, and Provean. The stability of the protein as the result of the mutation was studied using I- Mutant lastly the effect of the SNPs on the structure was predicted using Project hope.

\subsection{GeneMANIA}

(http://www.genemania.org) [8]. It is a web interface that finds other genes related to a set of input genes, using a very large set of functional association data. Gene name was entered into the software and the result show that an association data include protein and genetic interactions, pathways, coexpression, co-localization and protein domain similarity.

\subsection{SIFT (Sorting Intolerant from Tolerant) http://blocks.fhcrc.org/sift/SIFT.html [9]}

It is an online tool that predicts if an amino acid substitution affects protein function or not by using sequence homology. The dbSNP that were retrieved from NCBI were entered into the software and the result appears as deleterious or not according to whether amino acid substitution affects protein function based on sequence homology and the physical properties of amino acids.

\subsection{Polyphen-2 (Polymorphism Phenotyping v2)}

http://genetics.bwh.harvard.edu/pph2/. It is used to predict the possible impact of an amino acid substitution on both structure and function of protein by analysis of multiple sequence alignment and protein 3D structure [1]. The software estimates the position specific independent count score (PSIC) for every variant and then determines the difference between them, the higher the PSI, the higher the functional impact of the amino acid on the protein function may be. Prediction outcomes could be classified as probably damaging, possibly damaging or benign according to the score ranging from $(0-1)$.

\subsection{Provean (Protein Variation Effect Analyzer)}

(http://provean.jcvi.org/index.php). It is a software tool which predicts whether an amino acid substitution has an impact on the biological function of a protein. SNPs were entered using protein sequence. The Prediction outcomes could be classified as tolerated or deleterious.

\subsection{I-Mutant3.0}

(http://gpcr2.biocomp.unibo.it/cgi/predictors/I-

Mutant3.0/I-Mutant3.0.cgi For studying the effect of mutations on protein Stability. I- Mutant3.0 software was used.). It is a neural network based tool, predicts the change in the stability of the protein upon mutation [10]. The output is obtained in the form of protein stability change upon mutation and Gibbs-free energy change (DDG) either increased or decreased stability.

\subsection{Project Hope}

(http://www.cmbi.ru.nl/hope/). It is an automatic program that analyzes the structural and functional effects of point mutations. Five SNPs were inserted into the software and the results shows the effect of the mutation in the amino acid properties and how this affects the also plus an image for protein structure is displayed whenever available [10].

\subsection{SNPs \&GO (Single Nucleotide Polymorphism \&Gene Ontology), PHD-SNP}

(http://snps.biofold.org/snps-and-go) [2]. SNPs\& GO is an accurate method that, starting from a protein sequence, can predict whether a variation is disease related or not by exploiting the corresponding protein functional annotation. SNPs\& GO collects in unique framework information derived from protein sequence, evolutionary information, and function as encoded in the Gene Ontology terms, and outperforms other available predictive methods. [2] The protein sequences is submitted in FASTA format that is obtained from UniprotKB / ExPASY after submitting the sequence the mutations were submitted in the XPOSY format where $\mathrm{X}$ and $\mathrm{Y}$ are the wild-type and mutant residues respectively. The result is shown as Neutral or disease. PHDSNP results are presented as part of SNPs\& GO output

\section{Results}

In this study $A G T$ gene was found to have an association with 20 other different genes. Among them the most important one is $R E N$ (responsible for production of renin in the kidney) and AGTR2 (responsible for encoding receptor for angiotensin II) (Figure 1 and Table 1). The physical interaction and co expression of this gene with other related gene are shown in figure 1. The genes expressed with $A G T$ gene were shown in Table 3, Appendix.

The total number of SNPs obtained was 173 the nonsynonymous SNPs that were predicted to be deleterious by mutation were 46 and that not causing damage or tolerated were 127 SNPs using SIFT software. Analysis using Polyphen -2 revealed one SNP as benign, 12 as possibly damaging and 33 are probably damaging. Analysis with provean showed that 19 were neutral and 27 were deleterious. Protein stability was checked using I-mutant software which showsed13 SNPs increasing the protein stability and 33 were decreasing the protein stability. Prediction of whether SNPs were deleterious were checked using PhD-SNP software and showed 20 disease related and 18 were neutral in all SNPs. Also SNPs were checked using SNP \& Go software that showed 32 neutral and 14 were disease associated variation. The detailed results for SIFT, Polyphen-2, Provean-2 were shown in Table A1. The detailed results for I-mutant, SNP\&GO and PHD. 


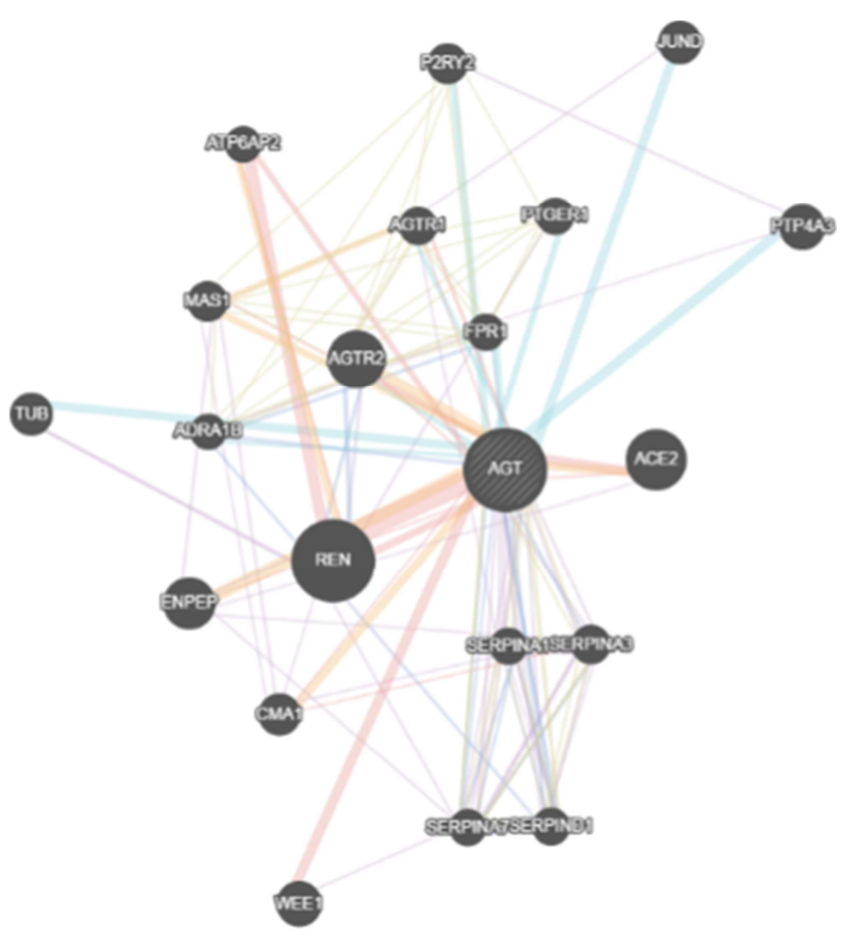

\section{Networks}

Physical Interactions

Co-expression

Predicted

Co-localization

Pathway

Genetic Interactions

Shared protein domains

Figure 1. GeneMANIA result.
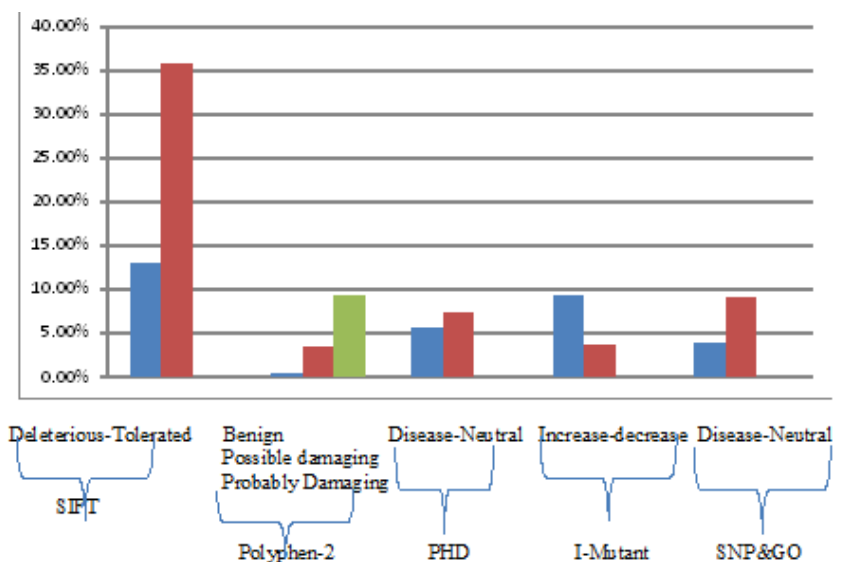

Figure 2. The results of different software.

Generally when using the sixth software (SIFT, Polyphen2, Provean, PhD, I-Mutant and SNP \& GO) all were showing abnormal appearance with effect as diseased, 33 SNPs showed decrease in protein stability using I-Mutant software. The structural impact of SNPs and its function was investigated using Project Hope software.

1. rs368513901, Tryptophan into a Arginine at position 261

The mutant residue is smaller than the wild-type residue. This will cause a possible loss of external interactions

The mutant residue is NEUTRAL, the mutant residue charge is POSITIVE. this can cause repulsion between the mutant residue and neighboring residues.

The wild-type residue is more hydrophobic than the mutant residue. The mutation might cause loss of hydrophobic interactions with other molecules on the surface of the protein.

2. rs137858911, Isoleucine into a Serine at position 345

The mutant residue is smaller than the wild-type residue. The mutation will cause an empty space in the core of the protein.

The wild-type residue is very conserved, but a few other residue types have been observed at this position too.

The wild-type residue is more hydrophobic than the mutant residue. The mutation will cause loss of hydrophobic interactions in the core of the protein.

3. rs137858911, Isoleucine into a Serine at position 345.

The mutant residue is smaller than the wild-type residue.

The mutation will cause an empty space in the core of the protein.

The hydrophobicity of the wild-type and mutant residue differs.

The mutation will cause loss of hydrophobic interactions in the core of the protein

4. rs375261929, Arginine into a Cysteine at position 458.

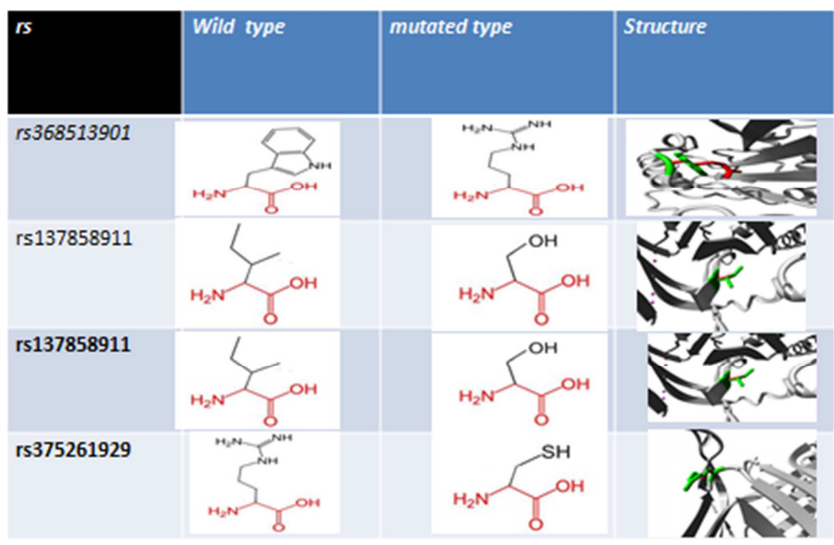

Figure 3. Project Hope results.

The mutant residue is smaller than the wild-type residue. The wild-type residue forms a hydrogen bond with Proline at position 347. The size difference between wild-type and mutant residue makes that the new residue is not in the correct position to make the same hydrogen bond as the original wild-type residue did.

The wild-type residue charge was POSITIVE, the mutant residue charge is NEUTRAL. The difference in charge will disturb the ionic interaction made by the original, wild-type residue.

The mutant residue is more hydrophobic than the wild- 
type residue. The difference in hydrophobicity will affect hydrogen bond formation. The wild-type residue forms a salt bridge with Aspartic Acid at position 352.

Results were shown in Figure 3.

\section{Discussion}

In this study all the 14 nsSNPs showed a damaging effect and disease related condition (rs143545998 (G29D), rs61731497 (C51R), rs2229389 (G114C), rs141302625 (L219Q), rs61762537 (R237C), rs145882750 (R237L), rs3685139 (W261R), rs56073403 (Y281C), rs147736976 (I345S), rs201501261 (P347L), rs137858911 (M381R), rs61762527 (P382A), rs375261929 (R458C), rs143479528 (P481L)). Five nsSNPs (rs61731497 (C51R), rs2229389 (G114C), rs61762537 (R237C) rs56073403 (Y281C) and rs61762527 (P382A), found in this study were also reported in another study as deleterious and causing disease in $A G T$ gene analysis using computational approach [12]. Also five nsSNPs (rs143545998 (G29D), rs141302625 (L219Q), rs145882750 (R237L), rs201501261 (P347L), and rs143479528 (P481L), were found to be deleterious in $A G T$ gene analysis [13]. The four remaining nsSNPs (rs368513901 (W261R), rs137858911 (M381R), rs147736976 (I345S) and rs375261929 (R458C) were reported in this study for the first time. The dominant clinical investigation was hypertension (NCBI) followed by renal tubular dysgenesis and eclampsia in human and mainly affecting the protein.

\section{Conclusions}

In conclusion, extensive functional and structural analyses are carried out to predict potentially damaging and deleterious nsSNPs of $A G T$ gene using bioinformatics and computational methods. In the study, 14 high confidence damaging nsSNPs are identified from 172 nsSNPs. Although bioinformatics tools have their limitations, the results from the present study may be convenient in future for further population based research activities and towards development of accuracy medicines.

\section{Recommendations}

SNPs in $A G T$ gene cause may diseases mainly hypertension and other related diseases for the fact that hypertension is a main chronic disease worldwide. More wetlab research regarding these 14 SNPs is recommended. The SNPs in the non-coding region also needs to be considere

\section{Appendix}

Table A1. SIFT, Provean and Polyphen-2 results.

\begin{tabular}{|c|c|c|c|c|c|}
\hline SNP & SIFT prediction & Provean score & Provean prediction & Polyphen-2 score & Polyphen-2 result \\
\hline rs4762 & Deleterious & -3.477 & Deleterious & 1 & Probably Damaging \\
\hline rs5041 & Deleterious & 0.157 & Neutral & 0.582 & Possibly Damaging \\
\hline rs 1805090 & Deleterious & -1.098 & Neutral & 0.852 & Possibly Damaging \\
\hline rs2229389 & Deleterious & -6.401 & Deleterious & 1 & Probably Damaging \\
\hline rs 11557882 & Deleterious & -1.572 & Neutral & 1 & Probably Damaging \\
\hline rs 11557883 & Deleterious & -3.898 & Deleterious & 0.55 & Possibly Damaging \\
\hline rs 11568032 & Deleterious & -1.411 & Neutral & 1 & Probably Damaging \\
\hline rs 17856352 & Deleterious & -6.039 & Deleterious & 0.685 & Possibly Damaging \\
\hline rs56073403 & Deleterious & -5.712 & Deleterious & 1 & Probably Damaging \\
\hline rs61731497 & Deleterious & -7.334 & Deleterious & 1 & Probably Damaging \\
\hline rs61751065 & Deleterious & -590 & Neutral & 1 & Probably Damaging \\
\hline rs61751067 & Deleterious & -1.776 & Neutral & 0.31 & Benign \\
\hline rs61751076 & Deleterious & -1.771 & Neutral & 1 & Probably Damaging \\
\hline rs61751077 & Deleterious & -2.798 & Deleterious & 0.998 & Probably Damaging \\
\hline rs61762537 & Deleterious & -5.296 & Deleterious & 1 & Probably Damaging \\
\hline rs 74315283 & Deleterious & -2.032 & Neutral & 1 & Probably Damaging \\
\hline rs 137858911 & Deleterious & -3.907 & Deleterious & 1 & Probably Damaging \\
\hline rs 140964843 & Deleterious & -1.747 & Neutral & 0.997 & Probably Damaging \\
\hline rs 141302625 & Deleterious & -5.332 & Deleterious & 0.975 & Probably Damaging \\
\hline rs 141724549 & Deleterious & -2.002 & Neutral & 1 & Probably Damaging \\
\hline rs 143437550 & Deleterious & -7.37 & Deleterious & 0.988 & Probably Damaging \\
\hline rs 143479528 & Deleterious & -7.37 & Deleterious & 1 & Probably Damaging \\
\hline rs 143545998 & Deleterious & -2.036 & Neutral & 1 & Probably Damaging \\
\hline rs 145882750 & Deleterious & -4.605 & Deleterious & 0.961 & Probably Damaging \\
\hline rs 146284519 & Deleterious & -4.053 & Deleterious & 1 & Probably Damaging \\
\hline rs 146566988 & Deleterious & -2.161 & Neutral & 1 & Probably Damaging \\
\hline rs 147355405 & Deleterious & -5.212 & Deleterious & 0.818 & Possibly Damaging \\
\hline rs 147736976 & Deleterious & -4.98 & Deleterious & 1 & Probably Damaging \\
\hline rs 149236456 & Deleterious & -1.452 & Neutral & 0.998 & Probably Damaging \\
\hline
\end{tabular}




\begin{tabular}{llllll}
\hline SNP & SIFT prediction & Provean score & Provean prediction & Polyphen-2 score & Polyphen-2 result \\
\hline rs149973083 & Deleterious & -1.102 & Neutral & 0.587 & Possibly Damaging \\
rs150161533 & Deleterious & -2.056 & Neutral & 0.799 & Possibly Damaging \\
rs151194891 & Deleterious & 0.328 & Neutral & 0.998 & Probably Damaging \\
rs199817559 & Deleterious & -0.666 & Neutral & 0.779 & Possibly Damaging \\
rs199864970 & Deleterious & -1.736 & Neutral & 0.648 & Possibly Damaging \\
rs200712921 & Deleterious & -2.908 & Deleterious & 0.997 & Probably Damaging \\
rs201162475 & Deleterious & -4.925 & Deleterious & 0.297 & Benign \\
rs201352496 & Deleterious & -2.638 & Deleterious & 1 & Probably Damaging \\
rs201501261 & Deleterious & -7.992 & Deleterious & 0.994 & Probably Damaging \\
rs201569036 & Deleterious & -1.366 & Neutral & 1 & Probably Damaging \\
rs267598410 & Deleterious & -3.579 & Deleterious & 0.603 & Possibly Damaging \\
rs368513901 & Deleterious & -3.579 & Deleterious & 1 & Probably Damaging \\
rs369727853 & Deleterious & -3.302 & Deleterious & 0.999 & Probably Damaging \\
rs374407232 & Deleterious & -0.307 & Neutral & 0.998 & Probably Damaging \\
rs374540090 & Deleterious & -3.019 & Deleterious & 0.824 & Possibly Damaging \\
rs375261929 & Deleterious & -4.844 & Deleterious & 0.982 & Probably Damaging \\
\hline
\end{tabular}

Table A2. I-mutant, SNP\& GO and PHD results.

\begin{tabular}{|c|c|c|c|c|}
\hline SNP & RI & I- mutant result & SNP\& GO & PHD \\
\hline rs4762 & 5 & Decrease & neutral & Neutral \\
\hline rs5041 & 7 & Decrease & neutral & Disease \\
\hline rs1805090 & 1 & Decrease & neutral & Neutral \\
\hline rs2229389 & 5 & Decrease & disease & disease \\
\hline rs11557882 & 4 & Decrease & neutral & neutral \\
\hline rs11557883 & 8 & Decrease & neutral & neutral \\
\hline rs11568032 & 9 & Decrease & neutral & disease \\
\hline rs17856352 & 7 & Increase & neutral & neutral \\
\hline rs56073403 & 4 & Decrease & disease & disease \\
\hline rs61731497 & 5 & Increase & disease & disease \\
\hline rs61751065 & 2 & Decrease & Neutral & neutral \\
\hline rs61751067 & 3 & Increase & Neutral & disease \\
\hline rs61751076 & 0 & Increase & Neutral & neutral \\
\hline rs61751077 & 1 & Decrease & Neutral & neutral \\
\hline rs61762527 & 8 & Decrease & Disease & disease \\
\hline rs61762537 & 1 & Increase & Disease & disease \\
\hline rs74315283 & 6 & Decrease & Neutral & neutral \\
\hline rs137858911 & 3 & Decrease & Disease & disease \\
\hline rs140964843 & 7 & Decrease & Neutral & neutral \\
\hline rs 141302625 & 4 & Decrease & Disease & disease \\
\hline rs 141724549 & 5 & Increase & Neutral & disease \\
\hline rs 143437550 & 7 & Decrease & Neutral & neutral \\
\hline rs 143479528 & 5 & Increase & Disease & disease \\
\hline rs 143545998 & 7 & Decrease & Disease & disease \\
\hline rs 145882750 & 2 & Increase & Disease & disease \\
\hline rs 146284519 & 5 & Decrease & Neutral & disease \\
\hline rs 146566988 & 2 & Increase & Neutral & neutral \\
\hline rs147355405 & 1 & Increase & Neutral & disease \\
\hline rs147736976 & 10 & Decrease & Disease & disease \\
\hline rs 149236456 & 5 & Decrease & Neutral & disease \\
\hline rs 149973083 & 9 & Decrease & Neutral & neutral \\
\hline rs150161533 & 8 & Decrease & Neutral & neutral \\
\hline rs151194891 & 9 & Decrease & Neutral & neutral \\
\hline rs 199817559 & 6 & Decrease & Neutral & disease \\
\hline rs 199864970 & 4 & Decrease & Neutral & disease \\
\hline rs200712921 & 1 & Decrease & Neutral & disease \\
\hline rs201162475 & 2 & Decrease & Neutral & disease \\
\hline rs201352496 & 8 & Decrease & Neutral & disease \\
\hline rs201501261 & 2 & Increase & Disease & disease \\
\hline rs201569036 & 2 & Increase & Neutral & neutral \\
\hline rs267598410 & 2 & Increase & Neutral & disease \\
\hline rs368513901 & 2 & Decrease & Disease & disease \\
\hline rs369727853 & 5 & Decrease & Neutral & neutral \\
\hline rs374407232 & 8 & Decrease & Neutral & neutral \\
\hline rs 374540090 & 7 & Decrease & Neutral & Disease \\
\hline rs375261929 & 5 & Decrease & Disease & Disease \\
\hline
\end{tabular}




\begin{tabular}{|c|c|c|}
\hline Gene & Description & Rank \\
\hline AGT & angiotensinogen [Source:HGNC Symbol;Acc:HGNC:333] & $\mathrm{N} / \mathrm{A}$ \\
\hline REN & renin [Source:HGNC Symbol;Acc:HGNC:9958] & 1 \\
\hline ACE2 & $\begin{array}{l}\text { angiotensin I converting enzyme } 2 \text { [Source:HGNC Symbol;Acc:HGNC: } \\
13557 \text { ] }\end{array}$ & 2 \\
\hline AGTR2 & angiotensin II receptor type 2 [Source:HGNC Symbol;Acc:HGNC:338] & 3 \\
\hline ENPEP & glutamyl aminopeptidase [Source:HGNC Symbol;Acc:HGNC:3355] & 4 \\
\hline PTP4A3 & $\begin{array}{l}\text { protein tyrosine phosphatase type IVA, member } 3 \text { [Source:HGNC } \\
\text { Symbol;Acc:HGNC:9636] }\end{array}$ & 5 \\
\hline WEE1 & WEE1 G2 checkpoint kinase [Source:HGNC Symbol;Acc:HGNC:12761] & 6 \\
\hline JUND & $\begin{array}{l}\text { JunD proto-oncogene, AP-1 transcription factor subunit [Source:HGNC } \\
\text { Symbol;Acc:HGNC:6206] }\end{array}$ & 7 \\
\hline TUB & $\begin{array}{l}\text { tubby bipartite transcription factor [Source:HGNC Symbol;Acc:HGNC: } \\
12406 \text { ] }\end{array}$ & 8 \\
\hline MA1 & chymase 1 [Source:HGNC Symbol;Acc:HGNC:2097] & 9 \\
\hline P2RY2 & purinergic receptor P2Y2 [Source:HGNC Symbol;Acc:HGNC:8541] & 10 \\
\hline MAS1 & $\begin{array}{l}\text { MAS1 proto-oncogene, G protein-coupled receptor [Source:HGNC } \\
\text { Symbol;Acc:HGNC:6899] }\end{array}$ & 11 \\
\hline EPINA3 & serpin family A member 3 [Source:HGNC Symbol;Acc:HGNC:16] & 12 \\
\hline AGTR1 & angiotensin II receptor type 1 [Source:HGNC Symbol;Acc:HGNC:336] & 13 \\
\hline PTGER1 & prostaglandin E receptor 1 [Source:HGNC Symbol;Acc:HGNC:9593] & 14 \\
\hline SERPIND1 & serpin family D member 1 [Source:HGNC Symbol;Acc:HGNC:4838] & 15 \\
\hline FPR1 & formyl peptide receptor 1 [Source:HGNC Symbol;Acc:HGNC:3826] & 16 \\
\hline SERPINA1 & serpin family A member 1 [Source:HGNC Symbol;Acc:HGNC:8941] & 17 \\
\hline SERPINA7 & serpin family A member 7 [Source:HGNC Symbol;Acc:HGNC:11583] & 18 \\
\hline ГP6AP2 & $\begin{array}{l}\text { ATPase } \mathrm{H}+\text { transporting accessory protein } 2 \text { [Source:HGNC Symbol;Acc: } \\
\text { HGNC:18305] }\end{array}$ & 19 \\
\hline
\end{tabular}

ADRA1B adrenoceptor alpha 1B [Source:HGNC Symbol;Acc:HGNC:278]

Figure A1. Genes co-expressed with AGT gene using GeneMANIA software.

Table A3. I-mutant, SNP\& GO and PHD results.

\begin{tabular}{llllll}
\hline Amino acid change & SNPs & Status & I-Mutant RI & I- Mutant result & SNPs\& GO \\
\hline G29D & rs143545998 & Reported & 7 & decrease & Disease \\
C51R & rs61731497 & Reported & 5 & increase & Disease \\
G114C & rs2229389 & Reported & 5 & decrease & Disease \\
L219Q & rs141302625 & Reported & 4 & decrease & Disease \\
R237C & rs61762537 & Reported & 1 & increase & Disease \\
R237L & rs145882750 & Reported & 2 & increase & Disease \\
W261R & rs368513901 & Novel & 2 & decrease & Disease \\
Y281C & rs56073403 & Reported & 4 & decrease & Disease \\
I345S & rs147736976 & Novel & 10 & decrease & Disease \\
P347L & rs201501261 & Reported & 2 & increase & Disease \\
M381R & rs137858911 & Novel & 3 & decrease & Disease \\
P382A & rs61762527 & Reported & 8 & decrease & Disease \\
R458C & rs375261929 & Novel & 5 & decrease & disease \\
P481L & rs143479528 & Reported & 5 & increase & disease \\
\hline
\end{tabular}




\section{References}

[1] Adzhubei I, Jordan DM, Sunyaev SR. Predicting functional effect of human missense mutations using PolyPhen-2. Curr Protoc Hum Genet. 2013; doi: 10.1002/0471142905.hg0720s.

[2] Calabrese R, Capriotti E, Fariselli P, Martelli PL, Casadio R. Functional annotations improve the predictive score of human disease-related mutations in proteins. Human Mutation. 2009; 30: $1237-1244$.

[3] Engeli, S., Sharma, A. The renin-angiotensin system and natriuretic peptides in obesity-associated hypertension. $J \mathrm{Mol}$ Med 79, 21-29 (2001).

[4] Edward A. Ross, Matthew J. Williams, Takashi Hamazaki, Naohiro Terada, William L. Clapp, Christopher Adin, Gary W. Ellison, Marda Jorgensen, and Christopher D. Batich. Embryonic Stem Cells Proliferate and Differentiate when Seeded into Kidney Scaffolds. J Am Soc Nephrol 20: 2338 2347, 2009. doi: 10.1681/ASN.2008111196

[5] Dodoo SN, Benjamin IJ. Genomic Approaches to Hypertension. Cardiol Clin. 2017 May; 35 (2): 185-196. doi: 10.1016/j.ccl.2016.12.001. Review.

[6] Gkaliagkousi E, Douma S, Zamboulis C, Ferro A. Nitric oxide dysfunction in vascular endothelium and platelets: role in essential hypertension. J Hypertens. 2009 Dec; 27 (12): 231020. doi: 10.1097/HJH.0b013e328330e89a. Review.

[7] Gribouval O, Gonzales M, Neuhaus T, Aziza J, Bieth E, Laurent N, Bouton JM, Feuillet F, Makni S, Ben Amar H, Laube G, Delezoide AL, Bouvier R, Dijoud F, OllagnonRoman E, Roume J, Joubert M, Antignac C, Gubler MC. Mutations in genes in the renin-angiotensin system are associated with autosomal recessive renal tubular dysgenesis. Nat Genet. 2005 Sep; 37 (9): 964-8. Epub 2005 Aug 14.

[8] Gubler MC, Antignac C. Renin-angiotensin system in kidney development: renal tubular dysgenesis. Kidney Int. 2010 Mar; 77 (5): 400-6. doi: 10.1038/ki.2009.423. Epub 2009 Nov 18. Review.

[9] gunda Padma, et al Risk conferred by tagged SNPs of AGT gene in causing susceptibility to essential hypertension, 2014, DOI: 10.3109/10641963.2014.881845.

[10] $\mathrm{Hu} \mathrm{J,} \mathrm{Ng} \mathrm{PC.} \mathrm{Predicting} \mathrm{the} \mathrm{effects} \mathrm{of} \mathrm{frame} \mathrm{shifting} \mathrm{indels}$ Genome Biol. 2012; vol. 13 pg. R9.

[11] Khalid Zuberi, Max Franz, Harold Rodriguez, Jason Montojo, Christian Tannus Lopes, Gary D. Bader Quaid Morr. GeneMANIA Prediction Server 2013 Update. Nucleic Acids Research. 2013; 41, Issue W1, 1 J W115-W122.

[12] mohamed al nagi et al Association of the angiotensinogen gene polymorphism with atherosclerosis and its risk traits in the Saudi population, 2013, Published online 2013 Mar 11. doi: 10.1186/1471-2261-13-17.

[13] pulakes purkait, Association of angiotensinogen gene SNPs and haplotypes with risk of hypertension in eastern Indian population, 2017, Published online 2017 Mar 29. doi: 10.1186/s40885-017-0069-x.

[14] Watkins WS, Hunt SC, Williams GH, Tolpinrud W, Jeunemaitre X, Lalouel JM, Jorde LB. Genotype-phenotype analysis of angiotensinogen polymorphisms and essential hypertension: the importance of haplotypes. J Hypertens. 2010 Jan; 28 (1): 65-75. doi: 10.1097/HJH.0b013e328332031a.

[15] Wolf G. Angiotensin II and tubular development. Nephrol Dial Transplant. 2002; 17 Suppl 9: 48-51. 